www.jmscr.igmpublication.org

Index Copernicus Value: 79.54

ISSN (e)-2347-176x ISSN (p) 2455-0450

crossref DOI: https://dx.doi.org/10.18535/jmscr/v7i6.185

Journal Of Medical Science And Clinical Research

IGM Publication

An Official Publication of IGM Publication

Original Article

\title{
Correlation of Absolute Neutrophil Count and Prevalence of Bacteremia in Chemotherapy Induced Neutropenia in a Tertiary Care Centre
}

\author{
Author \\ Dr Yazhini K, MBBS., MD \\ Department of Microbiology, Government Medical College, Thrissur, Kerala, India \\ Corresponding Author \\ Dr Yazhini K, MBBS., MD
}

Assistant Professor \& Consultant Microbiolgist, Meenakshi Ammal Dental College \& Meenakshi General Hospital

Address: No.12, Padmavathynagar, $13^{\text {th }}$ cross street, Thirumullaivoyal, Chennai -600062 , India

\begin{abstract}
Background: Neutropenic complications remain an important dose-limiting toxicity of cancer chemotherapy, associated with considerable morbidity, mortality, and cost. Bloodstream infection (BSI) is the most common complication leading to reduced dosage of chemotherapeutics and longer hospitalization. This study was aimed to determine the prevalence of BSI in neutropenic patients with solid tumours receiving chemotherapy in a tertiary care centre.

Objectives:

1. To determine the prevalence of bacteremia in neutropenicpatients with solid malignancy receiving chemotherapy.

2. To correlate the prevalence of bacteremia with the absolute neutrophil count of the patient.

Methods: A cross-sectional study was designed for prevalence of BSI in 150 patients with neutropenia who received chemotherapy for solid malignancies. Two samples of blood collected at one hour interval from each patient were plated onto agar plates and the isolates were identified by standard microbiological techniques and VITEK 2 identification systems.

Result: Among the 150 patients, 30(20\%) patients had microbiologically documented blood stream infection. Gram positive cocci contributed to $89 \%(n=28)$ and Gram negative bacilli 11\%(n=2). Among the Gram positive cocci, Staphylococcus epidermidis was the predominant isolate comprising $89.29 \%$ $(n=25)$.The patients with documented BSI had their Absolute neutrophil count level between $560 \times 109 / \mathrm{L}$ and $730 \times 109 / L$.

Conclusion: The prevalence of bloodstream infection in patients with chemotherapy induced neutropenia was $20 \%$ and Staphylococcus epidermidis was the predominant isolate (83.3\%). Hence, the Absolute neutrophil count below $1000 \times 109 / \mathrm{L}$ in patients receiving chemotherapy should alert the treating clinician about the possible risk of bloodstream infection to reduce hospitalization and to prevent the morbidity and mortatlity associated with neutropenia.
\end{abstract}

Keywords: Neutropenia, Chemotherapy, Bacteremia, Bloodstream infection, Chemotherapy induced neutropenia, Staphylococcus epidermidis. 


\section{Introduction}

Neutropenic complications remain an important dose-limiting toxicity of cancer chemotherapyassociated with considerable morbidity, mortality, and cost. ${ }^{1}$ Neutropenic patients are vulnerable to a wide spectrum of infectious agents which cause substantial mortality and morbidity among them. The increasing use of cytotoxic chemotherapy in patients with solid tumors has increased the number of patients who have neutropenia. ${ }^{2}$

Cytotoxic chemotherapy suppresses the hematopoietic system, impairing host protective mechanisms and limiting the doses of chemotherapy that can be tolerated. Neutropenia, the most serious hematologic toxicity, is associated with the risk of life-threatening infections as well as chemotherapy dose reductions and delays that may compromise treatment outcomes. ${ }^{3}$ In addition, neutropenia blunts the inflammatory response to nascent infections, allowing bacterial multiplication and invasion. $^{4}$

Bloodstream infection (BSI) by far is the most common complication in patients with cancer leading to delayed and reduced dosage of chemotherapeutics and longer hospitalization. Therefore, it contributes to suboptimal treatment, with higher morbidity and mortality rates. ${ }^{5-7}$

For the majority of chemotherapy regimens, the neutrophil count falls to its lowest level approximately 5-7 days after administration of chemotherapy and can take up to 2-4 weeks to recover. ${ }^{1}$ There is a tendency for neutropenic sepsis to occur more commonly in the first two cycles of treatment. ${ }^{8}$ Prior studies have shown that the risk of initial neutropenic events is greatest in the first cycle when most patients are receiving full-dose chemotherapy reporting $50-75 \%$ of initial neutropenic complications occurring within the first cycle. ${ }^{9}$ However, patients who have experienced an initial neutropenic event are at increased risk for additional neutropenic events during the subsequent treatment. ${ }^{10}$

Patients with solid tumours are a unique cohort; they frequently have implantable devices and are relatively immunocompromised, even without overt neutropenia. Surprisingly, only limited data have been reported on BSI in patients with solid tumors, in terms of the current epidemiology, etiology, and outcomes. ${ }^{11,12}$

To date, most information on BSI in patients with solid tumours has been extrapolated from studies of hematologic malignancies or studies that included both hematologic malignancies and solid tumours as a single group. ${ }^{13,14}$

The current prospective study involving hospitalized adults with BSI and solid tumours offers a comprehensive evaluation of epidemiology, and etiology exclusively in patients with BSI and solid tumours.

\section{Aims and Objectives}

1. To determine the prevalence of bacteremia in neutropenic patients with solid malignancy receiving chemotherapy.

2. To correlate the prevalence of bacteremia with the absolute neutrophil count of the patient.

\section{Material and Methods}

Study design: Cross sectional study

Sample size: 150

Sample size was calculated using the formula $\mathrm{n}=4 \mathrm{pq} / \mathrm{d}^{2}(\mathrm{p} \rightarrow$ prevalence, $\mathrm{q} \rightarrow 100-\mathrm{p}, \mathrm{d} \rightarrow$ allowable error) at significant level 0.05 and a power of $80 \%$.

\section{Study Population}

Adult patients between 18 to 65 years of age, who received chemotherapy for solid malignancies.

\section{Exclusion Criteria}

1. Patients who received antineoplastic chemotherapy for hematological malignancies.

2. Fungal causes of sepsis.

3. Neutropenia/neutropenic sepsis not caused by antineoplastic chemotherapy.

\section{Ethical Consideration}

Approval was obtained from the institutional ethical committee before the commencement of the study. Informed consent was obtained from the study population. All patients satisfying the 
inclusion criteria were documented. Patients were interviewed by structured questionnaire.

\section{Budget of the study}

The expenses were borne by the investigator and the institution. Study did not cause any additional financial burden on the patient.

\section{Study Procedure}

\section{Sample collection and processing:}

Under strict aseptic precautions, two samples of 5 $\mathrm{ml}$ blood at one hour interval were collected from the patients, added to bottles containing $45 \mathrm{ml}$ Brain-heart infusion broth and transported immediately to the laboratory in appropriate settings and sample processing done according to standard protocol. ${ }^{15}$

\section{Culture}

The brain-heart infusion broth containing the blood sample was incubated aerobically at $37^{\circ} \mathrm{C}$ and examined daily for 7 days. If turbidity or hemolysis was observed, subcultures were done onto Blood agar and Mac Conkey agar. The plates were incubated at $37^{\circ} \mathrm{C}$ aerobically and observed for growth every 24 hours for 48 hours.

\section{Identification of the isolates}

The isolates were identified based on colony morphology, ${ }^{16}$ Gram staining, motility, biochemical reactions by standard microbiological techniques and VITEK 2 identification systems.

\section{Controls}

The control organisms Escherichia coli ATCC 35218, Klebsiella pneumoniae ATCC 700603, Pseudomonas aeruginosa ATCC 27853, Acinetobacter baumannii ATCC BAA-747, Staphylococcus epidermidis ATCC 12228, Staphylococcus aureus ATCC 29213, Enterococcus faecalis ATCC 29212 were used to check the quality of media and reagents and to evaluate colour stability.

\section{Results}

Table 1: Distribution of Microbiologically documented BSI among patients with CIN

\begin{tabular}{|l|c|c|}
\hline $\begin{array}{l}\text { Microbiologically } \\
\text { documented BSI }\end{array}$ & $\begin{array}{c}\text { No. of } \\
\text { patients }\end{array}$ & Percentage \\
\hline Present & 30 & $20 \%$ \\
\hline Absent & 120 & $80 \%$ \\
\hline
\end{tabular}

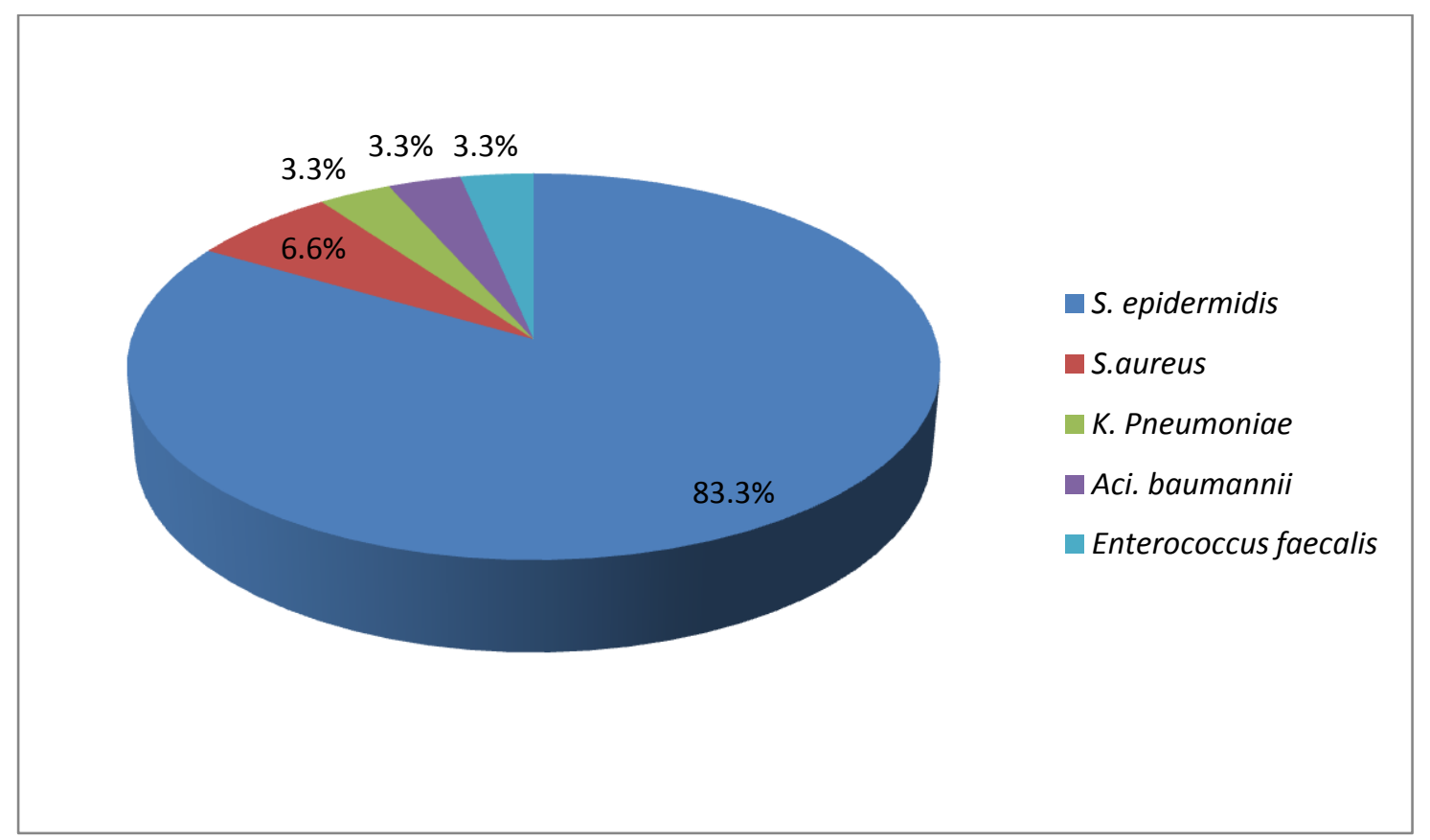

Figure 1: Comparative predominance etiological agents causing BSI in patients with CIN 


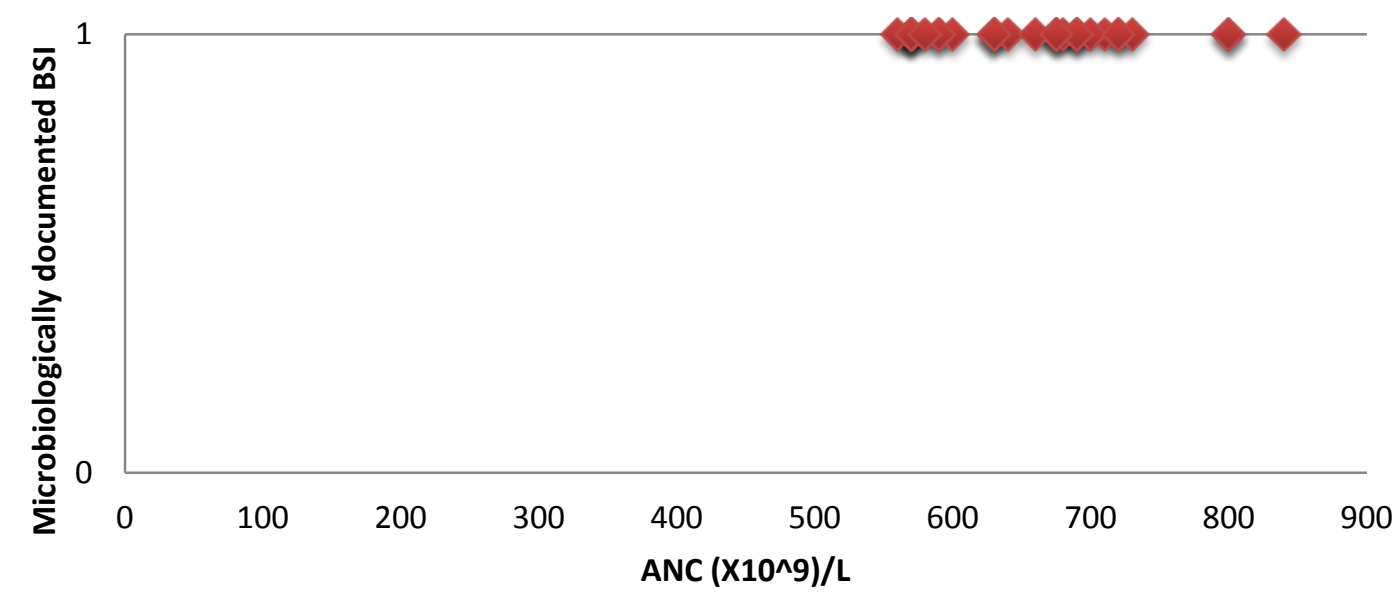

Figure 2: Correlation of incidence of BSI with ANC

\section{Discussion}

This study was undertaken in 150 patients and the study population included adults between 18 and 65 years of age. Microbiologically documented BSI was present in $20 \%(n=30)$ of the study group. A large majority of the patients with bacteremia had their ANC level between 560 $\times 10^{9} / \mathrm{L}$ and $730 \times 10^{9} / \mathrm{L}$ which shows that BSI in CIN is prevalent when the ANC falls below 730 $\mathrm{x} 10^{9} / \mathrm{L}$. Gram positive cocci contributed to $89 \%$ $(\mathrm{n}=28)$ and Gram negative bacilli $11 \%(\mathrm{n}=2)$. Staphylococcus epidermidis was the predominant isolate comprising $83.3 \%(\mathrm{n}=25)$.

Patients with Gram negative bacteremia have a poor prognosis and higher mortality. Hence all regimens are chosen to combat Gram negative sepsis. This may explain the shift of Gram negative bacteremia to Gram positive bacteremia.

Factors considered responsible for the shift towards Gram positive isolates, as discussed before, include aggressive chemotherapy and radiotherapy causing mucositis, profound and prolonged neutropenia, unrecognised herpetic infection of the mucous membrane, increased use of long indwelling intravenous catheters and use of newer quinolones in neutropenic patients.

A pilot study conducted among 1940 nonneutropenic control group over a period of one year in the same institution showed BSI with Staphylococcus epidermidis is prevalent only in
$3.60 \%(n=70)$ of the control population. Whereas, the prevalence of BSI with Staphylococcus epidermidis among 150 neutropenic patients in our study is $16.66 \%(\mathrm{n}=25)$, which is far higher than the prevalence in the control group. This justifies the role of Staphylococcus epidermidis as a potential pathogen in neutropenic patients.

\section{Conclusion}

The study on prevalence of bacteremia in chemotherapy induced neutropenia revealed some interesting insights into neutropenia and blood stream infections in Indian settings. Prevalence of bacteremia in patients with chemotherapy induce bacteremia was estimated to be $20 \%$. Staphylococcus epidermidis was the predominant isolate comprising $83.3 \%$ of the total isolates. The findings highlight the role of Staphylococcus epidermidis, which is commonly considered as a commensal, as a potential pathogen in neutropenic patients, and necessitate the inclusion of antimicrobials having gram positive coverage in the empirical antimicrobial therapy. The patients with documented bloodstream infection had their Absolute neutrophil count level between 560 $\times 10^{9} / \mathrm{L}$ and $730 \times 10^{9} / \mathrm{L}$, indicating a possible contrast in the risk of blood stream infections in chemotherapy induced neutropenia, between developed and developing countries like India. Hence, the Absolute neutrophil count below 1000 
$\times 10^{9} / \mathrm{L}$ in patients receiving chemotherapy should alert the treating Clinician about the possible risk of blood stream infection and sepsisto reduce hospitalization and to prevent the morbidity and mortatlity associated with neutropenia.

\section{Limitations of the Study}

1) The study was performed in a single institution, which may not reflect the epidemiology of different centres and/or different geographic areas.

2) Since anaerobic culture methods were not done, anaerobes could have been missed during the course of the study.

3) This study does not distinguish between community- and hospital- acquired infections.

4) The study was focussed on blood stream infections. Other foci of infection were not looked for in this study.

5) Several factors that might have influenced the epidemiology of causative pathogens and outcome in neutropenic patients with BSI (such as depth and duration of neutropenia; presence of lymphopenia; type and stage of underlying malignancy; and incidence, severity, and duration of mucositis) could not be assessed.

6) Clinical outcome could not be assessed as it was beyond the scope of this study.

\section{Declaration of Interest: None}

\section{References}

1. Holmes,

F.A., Jones,

S.E.,

O'Shaughnessy, J., Vukelja, S., George T, Savin, et al. (2002). "Comparable efficacy and safety profiles of once-percycle pegfilgrastim and daily injection filgrastim in chemotherapy-induced neutropenia: a multicenter dose-finding study in women with breast cancer." Annals of Oncology, 13(6), 9039.

2. Pizzo PA. Management of Fever in Patients with Cancer and Treatment- induced Neutropenia. N Engl J Med. 1993;328:1323-32.

3. Cancer 2004;100:228-37. (C) 2003 American Cancer Society.

4. National Cancer Institute. Common toxicity criteria, version 2.0. Available from

URL: http://ctep.cancer.gov/forms/CTCv20_430-992.pdf [access date January 3, 2003].

5. Freifeld AG, Bow EJ, Sepkowitz KA, Boeckh MJ, Ito JI, Mullen CA, Raad II, Rolston KV, Young JA, Wingard JR. Infectious Diseases Society of America (2011) clinical practice guideline for the use of antimicrobial agents in neutropenic patients with cancer: 2010 update by the Infectious Diseases Society of America. Clin Infect Dis. 2011; 52: 427-431.

6. Klastersky J. Management of fever in neutropenic patients with different risks of complications. Clin Infect Dis. 2004; 39: S327.

7. Montassier E, Batard E, Gastinne T, Potel $\mathrm{G}$, de la Cochetiere MF. Recent changes in bacteraemia in patients with cancer: a systematic review of epidemiology and antibiotic resistance. Eur J ClinMicrobiol Infect Dis. 2013; 32: 841-850.

8. Culakova, E., Thota, R., Poniewierski, M.S., Kuderer, N.M., Wogu, A.F., Dale, D.C., Crawford, J. and Lyman, G.H. (2014). "Patterns of chemotherapyassociated toxicity and supportive care in US oncology practice: a nationwide prospective cohort study." Cancer Medicine, 3(2), 434-44.

9. Crawford J, Dale DC, Kuderer NM, Culakova E, Poniewierski MS, Wolff D, et al. Risk and timing of neutropenic events in adult cancer patients receiving chemotherapy: the results of a prospective nationwide study of oncology practice. J. Natl. Compr. Canc. Netw. 2008;6:109118. 
10. Silber JH, Fridman M, DiPaola RS, Erder MH, Pauly MV, Fox KR. First-cycle blood counts and subsequent neutropenia, dose reduction, or delay in early-stage breast cancer therapy. J. Clin. Oncol. 1998;16:2392-2400.

11. Anatoliotaki M, Valatas V, Mantadakis E, Apostolakou H, Mavroudis D, Georgoulias V, Rolston KV, Kontoyiannis DP, Galanakis E, Samonis G. Bloodstream infections in patients with solid tumors: associated factors, microbial spectrum and outcome. Infection.2004; 32: 65-71.

12. Schelenz S, Nwaka D, Hunter PR. Longitudinal surveillance of bacteraemia in haematology and oncology patients at a UK cancer centre and the impact of ciprofloxacin use on antimicrobial resistance. J AntimicrobChemother.2013; 68: 1431-1438.

13. Gudiol C, Bodro M, Simonetti A, Tubau F, Gonzalez-Barca E, Cisnal M, Domingo-Domenech E, Jimenez L, Carratala J. Changing aetiology, clinical features, antimicrobial resistance, and outcomes of bloodstream infection in neutropenic cancer patients. Clin Microbiol Infect. 2013: 474-479. Epub 2012 Apr 24.

14. Wisplinghoff $H$, Seifert H, Wenzel RP, Edmond MB. Current trends in the epidemiology of nosocomial bloodstream infections in patients with hematological malignancies and solid neoplasms in hospitals in the United States. Clin Infect Dis. 2003; 36: 1103-1110.

15. Mackie, T.J. and McCartney, J.E. Practical medical microbiology.14th edition. Churchill Livingstone, New York; 1996.

16. Betty A. Forbes ,Daniel.F.Sahm, Alice S. Weissfeld Bailey \& Scott's Diagnostic Microbiology $12^{\text {th }}$ edition. 